\title{
Estudo clínico-epidemiológico da cardiomiopatia de Takotsubo em um hospital de referência em Fortaleza, Ceará, Brasil
}

Clinical-epidemiological study of Takotsubo cardiomyopathy at a reference hospital in Fortaleza, Ceará, Brazil

Estudio clínico-epidemiológico de la cardiomiopatía de Takotsubo en un hospital de referencia en Fortaleza, Ceará, Brasil

\author{
Márcio da Silva PEREIRA ${ }^{1}$ \\ Ana Maria Viola RENSI ${ }^{2}$ \\ Silvana Céspedes GóMEZ ${ }^{3}$ \\ Roberto Eduardo Donoso RIVERO 4 \\ Júlio de Oliveira PORTES ${ }^{5}$ \\ Marcelo Rocha SCARAMUSSA ${ }^{6}$ \\ Ana Flávia Moura MENDES ${ }^{7}$ \\ Ismael Geraldo de LIMA ${ }^{8}$ \\ Michaella Fernandes ALENCAR ${ }^{9}$ \\ Maria Rita Carvalho de Freitas AMORIM ${ }^{\mathbf{1 0}}$
}

\author{
${ }^{1}$ Acadêmico de Medicina do Centro Universitário Christus (UNICHRISTUS), 60190-060, Fortaleza - Ceará, Brasil. \\ ${ }^{2}$ Médica graduada pela Universidade Brasil (UB), 15600-000, Fernandópolis - São Paulo, Brasil. \\ ${ }^{3}$ Médica do Centro de Saúde Escola Barra Funda (CSEBF), 01133-020, São Paulo-SP, Brasil. \\ ${ }^{4}$ Médico graduado pela Universidad Autónoma Gabriel René Moreno (UAGRM), 10260, Santa Cruz de la Sierra, Bolívia. \\ ${ }_{5}^{5}$ Acadêmico de medicina da Universidade Federal de São Joao Del Rei (UFSJ), 36301-360, Divinópolis - Minas Gerais, Brasil. \\ ${ }^{6}$ Médico graduado pela Universidade de Itaúna (UIT), 35680-142,Itaúna-Minas Gerais, Brasil. \\ ${ }^{7}$ Médica graduada pela Universidade José do Rosário Vellano (UNIFENAS), 31270-020, Belo Horizonte - Minas Gerais, Brasil. \\ ${ }^{8}$ Médico graduado pela Universidade Presidente Antônio Carlos (UNIPAC), 36048-000, Juiz de Fora - Minas Gerais, Brasil. \\ ${ }^{9}$ Médica graduada pela Universidade Federal do Pernambuco (UFPE), 50670-901, Recife - Pernambuco, Brasil. \\ ${ }^{10}$ Médica graduada pela Universidade Potiguar (UNP), 59020-010, Natal-Rio Grande do Norte
}

\section{Resumo}

A Cardiomiopatia de Takotsubo (CMT), também denominada Síndrome do Coração Partido, constitui uma entidade nosológica própria e uma causa rara de disfunção transitória do ventrículo esquerdo, na ausência de doença coronária significativa. O propósito do presente trabalho foi analisar as características clínico-epidemiológicas dos casos internados por Cardiomiopatia de Takotsubo no Hospital de Messejana (HM), explorando a possível relação entre um estressor específico e o desenvolvimento da CMT. Trata-se de estudo epidemiológico descritivo, prospectivo, com eixo transversal analítico no qual foram observados prospectivamente todos os prontuários de pacientes com diagnóstico presumido de IAM e circunscreveu-se o tamanho da amostra aos casos com diagnóstico confirmado de Cardiomiopatia de Takotsubo, segundo os critérios de 2004 de Bybee et al. Os resultados apontaram que de 1147 prontuários analisados, entre janeiro e agosto de 2016, 9 pacientes satisfizeram os critérios para o diagnóstico de CMT. Oito $(88,8 \%)$ pacientes eram do sexo feminino, com idade média de 60,7 anos. Dos pacientes analisados apenas $4(44,4 \%)$ tinham estressores emocionais associados. Identificou-se forte associação entre idade e fator estressor prévio $(r=0,799)$. Concluiu-se que a Cardiomiopatia de Takotsubo deve sempre ser considerada no diagnóstico diferencial da Síndrome coronariana aguda, mesmo os sintomas não tendo sido precipitados por intenso estresse emocional.

Descritores: Cardiomiopatia de Takotsubo; Disfunção Ventricular; Miocárdio Atordoado.

\begin{abstract}
Takotsubo Cardiomyopathy (TCM), also called the Broken Heart Syndrome, constitutes a nosologic entity of its own and is a rare cause of transient left ventricular dysfunction in the absence of significant coronary disease. The objective of this paper was analyzed the clinical and epidemiological characteristics of patients hospitalized for Takotsubo Cardiomyopathy at the Hospital de Messejana (HM), exploring the possible relationship between a specific stressor and the development of Takotsubo Cardiomyopathy. This is a descriptive, prospective epidemiological study with an analytical transversal axis in which all medical records of patients with a suspected AMI diagnosis were prospectively analyzed and the sample size circumscribed to cases with a confirmed diagnosis of Takotsubo Cardiomyopathy, according to the criteria of Bybee et al. at 2004. The results pointed that from 1147 patient charts analyzed, between January and August 2016, 9 patients met the criteria for the diagnosis of CMT. Eight (88.8\%) patients were female, with a mean age of 60.7 years. Of the patients analyzed, only $4(44.4 \%)$ had associated emotional stressors. It was identified a strong association between age and previous stressor $(r=0.799)$. It is concluded that Takotsubo Cardiomyopathy should always be considered in the differential diagnosis of acute coronary syndrome, even if the symptoms were not precipitated by intense emotional stress.

Descriptors: Takotsubo Cardiomyopathy; Ventricular Dysfunction; Myocardial Stunning.

\section{Resumen}

La Cardiomiopatía de Takotsubo, también denominada Síndrome del Corazón del Partido, constituye una entidad nosológica propia y una causa rara de disfunción transitoria del ventrículo izquierdo, en ausencia de una enfermedad coronaria significativa. El objetivo del presente trabajo fui analizar las características clínico-epidemiológicas de los casos internados por Cardiomiopatía de Takotsubo en el Hospital de Messejana (HM), explorando la posible relación entre un estresante específico y el desarrollo de la CMT. Trata-se de estudio epidemiológico descriptivo, prospectivo, con eje transversal analítico. Se observó prospectivamente todos los prontuarios de pacientes con diagnóstico presumido de IAM y se circunscribió el tamaño de la muestra a los casos con diagnóstico confirmado de Cardiomiopatía de Takotsubo, según los criterios de Bybee et al. do año de 2004. Resultados mostraran que de 1147 prontuarios analizados, entre enero y agosto de 2016, 9 pacientes cumplieron los criterios para el diagnóstico de CMT. Ocho $(88,8 \%)$ pacientes eran del sexo femenino, con edad media de 60,7 años. De los pacientes analizados sólo $4(44,4 \%)$ tenían estresores emocionales asociados. Se identificó una fuerte asociación entre edad y factor estresante previo $(r=0,799)$. Concluí-sé que la Cardiomiopatía de Takotsubo debe siempre ser considerado en el diagnóstico diferencial del Síndrome coronario agudo, incluso los síntomas no habiendo sido precipitados por intenso estrés emocional. Descriptores: Cardiomiopatía de Takotsubo; Disfunción Ventricular; Aturdimiento Miocárdico.
\end{abstract}

\section{INTRODUÇÃO}

A disfunção ventricular induzida pelo estresse, também conhecida como Cardiomiopatia de Takotsubo
(CMT), broken heart syndrome ou apical ballooning syndrome constitui uma entidade nosológica própria e uma 
causa rara de aneurisma ventricular esquerdo agudo, na ausência de doença coronária significativa ${ }^{1}$. Caracteriza-se por uma disfunção transitória do ventrículo esquerdo, de caráter reversível, induzida por um estresse físico ou psíquico, que mimetiza uma síndrome coronariana aguda, podendo até culminar em choque cardiogênico (Figura 1). Recebeu a designação de Takotsubo devido à forma peculiar adquirida pelo ventrículo esquerdo, no ventriculograma, no final da sístole, comparando-a ao típico vaso (termo em japonês Tsubo) usado na pesca do polvo (termo em japonês Tako $)^{1,2}$.
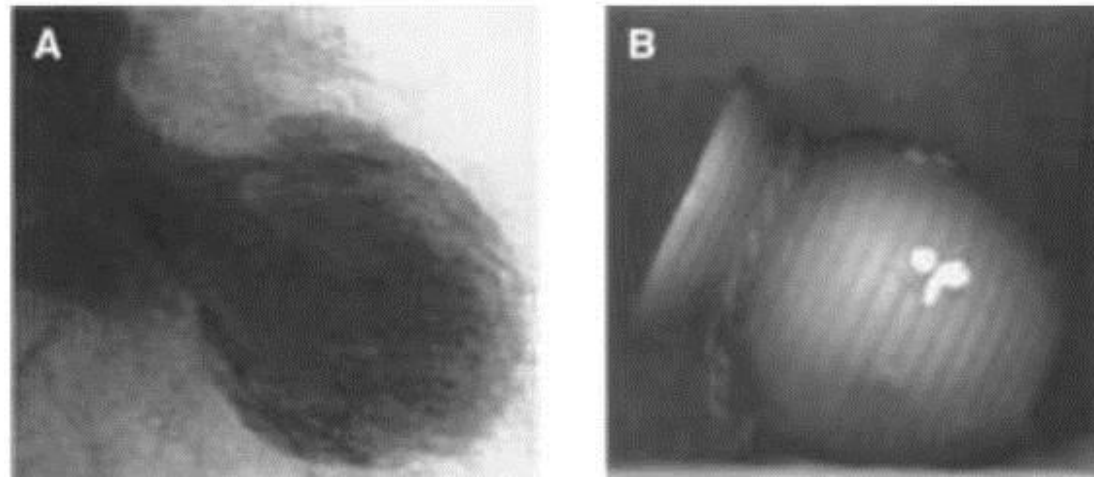

Figura 1: (A) Ventrículo esquerdo com o aspecto apical ballooning na ventriculografia; (B) Armadilha de Octopus retirado de Prasad et al. ${ }^{2}$.

Segundo Kawai et al. ${ }^{3}$ essa doença afeta pelo menos $1-2 \%$ dos pacientes admitidos com alterações eletrocardiográficas agudas do segmento $\mathrm{ST}^{3}$. No início, acreditava-se que era exclusiva da população japonesa, onde foi originalmente descrita na década de noventa. No entanto, na última década, vários casos têm sido relatados em todo o mundo. Nos Estados Unidos, por exemplo, 2-2.2\% dos pacientes admitidos com quadro clínico típico de uma Síndrome Coronariana Aguda foram posteriormente diagnosticados com a Síndrome de Takotsubo ${ }^{5}$.

Outro estudo de prevalência conduzido por Park et al. ${ }^{6}$ evidenciou que um terço dos pacientes internados em uma unidade de terapia intensiva com diagnóstico prévio de doença não cardíaca (insuficiência respiratória e sepse), eram, na verdade, portadores da Síndrome de Takotsubo. Sexo feminino e idade avançada foram os principais fatores de risco reconhecidos, e $90 \%$ dos casos foram descritos em mulheres na pós-menopausa ${ }^{6}$.

Várias diretrizes (guidelines) surgiram para tornar a Cardiomiopatia de Takotsubo amplamente reconhecida, bem como também, para melhor definir os critérios diagnósticos. Segundo a mais recente, proposta pela Clínica Mayo (EUA) e pelo Takotsubo Cardiomyopathy Study Group (Japão), a Cardiomiopatia de Takotsubo pode ser definida pelos seguintes critérios: (1) hipocinesia, discinesia ou acinesia transitória dos segmentos médios e apicais do ventrículo esquerdo (VE) com ou sem envolvimento apical, com alterações da contratilidade da área de vascularização correspondente a mais do que uma artéria coronária; (2) ausência de doença coronariana obstrutiva ou angiografia que evidencie uma ruptura de placa arteriosclerótica; (3) alterações eletrocardiográficas de novo (supradesnivelamento de ST e/ou inversão das ondas T) ou aumento discreto dos níveis séricos de troponina; (4) ausência de feocromocitoma ou miocardite. Vale ressaltar que a diretriz japonesa designa o balonamento apical secundário, acidentes cerebrovasculares e feocromocitomas como "Disfunções MiocárdicasTakotsubo-like",

A fisiopatologia da doença ainda não foi totalmente elucidada, no entanto, várias hipóteses concordam que o excesso de catecolaminas (gerado por atuação medicamentosa ou, principalmente, situações de estresse) tenha um papel decisivo na patogênese da doença. Elas são responsáveis por gerar espasmo microvascular, hipocontratilidade, o que causa dano ao músculo cardíaco, afetando principalmente o ápice do coração, pois nessa área predominam receptores $\beta$-adrenérgicos. Modelos com ratos demonstraram que o excesso de catecolaminas é responsável por um efeito inotrópico negativo, como pode ser explicado por um detalhado experimento proposto por Lyon et al. ${ }^{8}$ Esse modelo demonstrou que a interrupção do tráfico de sinal intracelular pela proteína Gs à proteína $\mathrm{Gi}$, via sinalização de receptores $\beta 2$-adrenérgicos, leva a um inotropismo negativo. Portanto, variações na quantidade de receptores de indivíduo para indivíduos explicariam a variação dos quadros na síndrome ${ }^{9,10}$.

Além disso, essa disfunção microvascular, causada provavelmente pelas catecolaminas, foi também sugerida por Elesberetal (2006), quando foi relatado um grau de perfusão TIMI anormal do miocárdio (um índice angiográfico da perfusão miocárdica) em $69 \%$ de seus pacientes com a síndrome de takotsubo. Contudo, ainda não foi claramente definido se a disfunção microvascular é um fenômeno primário ou secundário ${ }^{11}$.

$\mathrm{O}$ aumento da incidência de DPOC e asma brônquica (patologias em que há aumento das catecolaminas) foi encontrado por Herttinget al em 32 pacientes diagnosticados retrospectivamente com takotsubo, logo, este resultado somado aos outros estudos nos quais as catecolaminas plasmáticas foram medidas durante a apresentação reforçam essa hipótese neurohormonal ${ }^{12}$.

Perante esse caráter agudo, transitório e de certa forma influenciado pelo meio ambiente, têm sido propostos vários outros mecanismos fisiopatológicos que se encontram ainda em investigação: ocorrência de espasmos coronários; isquemia microvascular; cardiotoxicidade às catecolaminas; rutura de placa isolada na artéria coronária descendente anterior; e obstrução aguda e dinâmica do trato de saída do VE, miocardite aguda (contudo, alterações estruturais e ultraestruturais do miocárdio são mais relacionadas à toxidade direta das catecolaminas) e o envolvimento hormonal, principalmente do estrógeno, pois, como foi descrito, a maioria dos casos ocorre com mulheres na pós-menopausa, destacando o papel protetor cardiovascular do estrógeno ${ }^{11}$.

O prognóstico é geralmente favorável com recuperação completa de todas as alterações que caracterizam a Cardiomiopatia de Takotsubo, normalmente até duas a quatro semanas. Não existe tratamento específico, dada a reversibilidade e a incerteza fisiopatológica. Deve optar-se por terapêutica de suporte, de acordo com o grau de disfunção sistólica e direcionada para as complicações agudas, que ocorrem em cerca de $20 \%$ dos doentes, variando amplamente desde choque cardiogênico, edema agudo do pulmão, arritmias, formação de trombos ao nível do VE com eventual embolia até à morte?

Pouco é conhecido sobre os mecanismos etiológicos para as anormalidades de motilidade da parede cardíaca características da Síndrome de Takotsubo; no entanto, essa cardiomiopatia parece ter uma predileção por mulheres idosas e é classicamente associada a um severo gatilho emocional, psicológico ou físico, levando ao balonamento apical da parede cardíaca e aos sintomas clínicos de infarto agudos do miocárdio ${ }^{3}$. Portanto, o presente estudo se propõe a determinar se esse fator estressor visto na Cardiomiopatia de Takotsubo (CMT) é um fator determinante para desencadeamento da síndrome. Pretende-se ainda analisar as características clínicas dos casos internados por CMT no 
Hospital de Messejana (HM), explorarando a possível relação entre um estressor específico e o desenvolvimento da CMT.

\section{MATERIAL E MÉTODO}

Realizou-se um estudo epidemiológico descritivo, prospectivo, com eixo transversal analítico. A pesquisa foi desenvolvida no setor de parada cardiorrespiratória do Hospital de Messejana Dr. Carlos Alberto Studart Gomes, referência em assistência no estado do Ceará.

Para se identificar a população do estudo, foram observados prospectivamente todos os prontuários físicos de pacientes com diagnóstico presumido de Infarto Agudo do Miocárdio (IAM) expedidos pelo setor de parada cardiorrespiratório do Hospital de Messejana, admitidos sequencialmente no período compreendido entre janeiro e agosto de 2016 (por razões de ordem logística - facilidade de acesso aos dados e exiguidade de tempo útil para proceder à pesquisa de dados de todos os casos). Circunscreveu-se o tamanho da amostra dos casos suspeitos de IAM da parede anterior com elevação de ST aos pacientes com diagnóstico confirmado de Cardiomiopatia de Takotsubo, segundo os critérios de Bybee et al. ${ }^{13}$. Relativamente aos casos de IAM da parede anterior foram utilizados como critérios de inclusão os propostos pela Sociedade Europeia de Cardiologia para o diagnóstico de infarto (todos cumpridos e observáveis nos processos clínicos) e os seguintes critérios de supra desnivelamento de ST: supra desnivelamento do segmento ST desde o ponto J $\mathrm{de} \geq 0,2 \mathrm{mV}$ nas derivações pré-cordiais $\mathrm{V} 2$ e $\mathrm{V} 3 \mathrm{e} \geq 0,1 \mathrm{mV}$ nas restantes ${ }^{11}$.

Foram incluídos no estudo, pacientes com idades compreendidas entre 18 e 90 anos que possuíam o diagnóstico confirmado de Cardiomiopatia de Takotsubo segundo critérios de Bybee et al. ${ }^{13}$ para cardiomiopatia induzida pelo estresse e que portanto tivessem: alterações do ECG (elevação de ST ou ondas T invertidas) na fase aguda da sintomatologia; realizado cateterismo na fase aguda que tenha revelado ausência de doença coronária significativa; presença de concentrações plasmáticas elevadas dos marcadores de necrose miocárdica; ventriculografia e/ou ecocardiografia com demonstração de acinesia ou discinesia transitórias dos segmentos apicais e médio ventriculares associados com alterações regionais da motilidade parietal que se estendessem para além da distribuição do fluxo sanguíneo na dependência de um único vaso coronário epicárdico ${ }^{13}$.

Foram excluídos do estudo os prontuários que apresentaram predominante ausência de dados, como aqueles que constaram apenas o diagnóstico clínico de Cardiomiopatia ou casos de óbitos que não passaram pelas avaliações dos profissionais da instituição.

Os dados foram coletados dos formulários de admissão, das fichas de avaliação e de evolução e dos relatórios de alta. $\mathrm{O}$ registro das informações obtidas foi realizado em formulário próprio desenvolvido para a pesquisa, em que foram registrados dados referentes às variáveis demográficas, fatores de risco cardiovascular, forma de apresentação, fatores desencadeantes (estresses anteriores), bioquímica, complicações, resultados eletrocardiográficos, ecocardiográficos e cateterismo cardíaco na fase aguda da doença, bem como também o desfecho clínico desses doentes.

As variáveis definidas no estudo foram organizadas em planilhas do programa Excel® versão 2016, Microsoft
Corporation, EUA. Os dados obtidos na coleta foram distribuídos nas planilhas, em colunas numeradas e intituladas pelas variáveis descritas, a fim de permitir a análise estatística dos mesmos. Para a descrição das variáveis quantitativas foram utilizadas estatísticas descritivas, como a média, desvio padrão, mediana, o mínimo e o máximo.

Utilizou-se para a realização dos cálculos estatísticos o programa Statistical Package for Social Sciences (SPSS), versão 17.0. Os resultados obtidos nas análises estatísticas foram dispostos em tabelas. Na análise estatística descritiva foi verificada a distribuição da Cardiomiopatia de Takotsubo pela freqüência das variáveis: idade, sexo, região de origem, profissão, renda familiar, escolaridade, fatores de risco.

Para análise dos dados, foram obtidas distribuições de frequências absolutas e percentuais e as medidas estatísticas: média, desvio-padrão e intervalo de confiança.

Para as variáveis quantitativas, foram utilizados testes paramétricos como o teste $\mathrm{T}$ de student, análise de variância (ANOVA) e teste de Mann-whitney e dentre os teste não paramétrico, utilizou-se o teste de Bartlett.

Para comparação das variáveis nominais, foi aplicado o teste Qui-Quadrado. Para realizar a associação entre as variáveis contínuas, foi realizada a correlação linear de Pearson e para as descontínuas, o de Spearman. Nas correlações definidas, foram considerados valores significativos para $\mathrm{p}<0,05$, com IC $95 \%$.

$\mathrm{O}$ projeto do estudo em questão foi submetido ao Comitê de Ética em Pesquisa do Centro Universitário Christus. O Centro de Estudos e Pesquisa do Hospital de Messejana foi informado sobre o estudo, recebendo uma cópia do projeto de pesquisa e um termo solicitando a autorização da pesquisa. A concordância aos termos expostos no projeto e a aprovação pelo Centro de Estudos foi efetivada mediante a assinatura do Superintendente Técnico da instituição no parecer consubstanciado fornecido pelo setor responsável do hospital.

\section{RESULTADOS E DISCUSSÃO}

De 1147 prontuários analisados de pacientes com diagnóstico presumido de infarto agudo do miocárdio, entre janeiro e agosto de 2016, 9 (90\%) pacientes satisfizeram os critérios para o diagnóstico de $\mathrm{CMT}$, dos quais 8 eram eram do sexo feminino e 1 do sexo masculino. A idade média foi de 60,7 anos (95\% IC, 42 - 78). Oito (88,9\%) pacientes apresentaram como principal manifestação clínica a dor no peito. Dispneia esteve presente em 6 pacientes $(66,7 \%)$. Dos pacientes analisados apenas $4(44,4 \%)$ tinham estressores emocionais associados, dois deles por morte trágica (assassinato de entes queridos); um caso de guarda de menor de idade e o outro tinha relato que vinha sofrendo de quadro depressivo a algumas semanas. Outros possíveis fatores causais observados foram: eventos iatrogênicos (ecocardiografia de estresse com uso de dobutamina e por associação de anti-histamínico e pseudoefedrina) e quadro de sepse de origem hospitalar (pielolitotomia complicada por sepse 3 semanas antes dos sintomas e infecção por Pseudomonas aeruginosas adquirida durante internamento hospitalar).

Em nosso estudo pudemos constatar correlação estatisticamente significativa entre idade e fator estressor prévio aplicando-se o coeficiente de correlação linear de Pearson $(r=0,799)$, conforme apresentado no Gráfico 1. Dessa forma, quanto menor a idade maior a possibilidade de 
associação com algum evento estressor prévio.

Os achados mais comuns do ECG foram: prolongamento do intervalo QT $(\mathrm{n}=2)$; alterações inespecíficas de ST $(n=3)$ e elevação do segmento ST $(n=7)$. A média dos níveis de troponina foi de 2,244 $(0,4-4,5)$ e a média de CK-MB de 20,22 (3,0 - 42,0). Oito $(88,9 \%)$ pacientes apresentavam níveis de troponina que preencheram os critérios para IAM em nossa instituição $(>0.5 \mathrm{ng} / \mathrm{ml})$ e sete preencheram critérios pela CK-MB $(>5 \mathrm{ng} / \mathrm{ml})$.

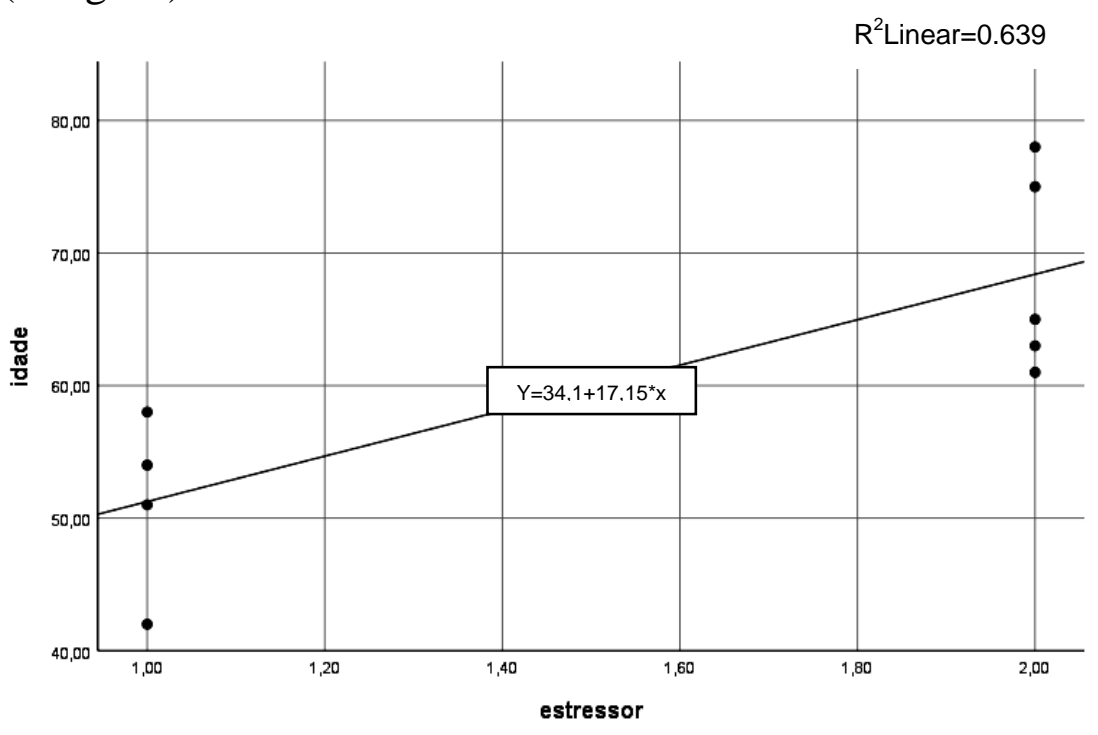

Grafico 1: Disperso simples de idade por estressor.

Todos os pacientes do estudo foram submetidos ao cateterismo cardíaco, que evidenciou hipercontratilidade basal em todos os casos associado à acinesia apical-medial em $7(77,7 \%)$ casos, acinesia apical em $4(44,4 \%)$ casos e acinesia ântero-apical em $2(22,2 \%)$ caso. Em todos os casos ficou evidente o balonamento aneurismático do ventrículo esquerdo.

As comorbidades mais frequentes foram hipertensão arterial sistêmica (3 pacientes - 33,3\%), diabetes tipo 2 (5 pacientes - 55,6\%), dislipidemia (2 pacientes $-22,2 \%)$ e tabagismo ( 2 pacientes - 22,2\%) com carga maior que 20 maços/ano).

Traçando um perfil dos pacientes portadores de CMT do nosso estudo, poderíamos afirmar que se trata de uma doença que acomete majoritariamente mulheres na pósmenopausa admitidas no pronto socorro com um quadro de dor pré-cordial associada ou não a evento estressor prévio.

Apesar da maioria dos estudos prévios apontarem para associação da CMT com um evento estressor, pudemos observar que apenas $44,4 \%$ dos casos (4 pacientes) tiveram um fator estressor claramente identificado como gatilho desencadeante da síndrome. Mais da metade dos pacientes não tiveram fatores estressores identificáveis nem mesmo durante sua estadia no hospital.

É difícil se determinar causalidade em estudos prospectivos. Dessa forma, a nossa série de casos, de caráter prospectivo, deve ser vista como um dos estudos piloto elucidativo quanto aos fatores causais dessa síndrome rara.

Espera-se que $o$ fator estressante preceda o aparecimento da CMT, mas o intervalo entre ele e o aparecimento dos sintomas é desconhecido. Em um estudo investigando ECGs armazenados no marcapasso dos pacientes em Nova York e em Nova Jersey antes e depois dos atentados do World Trade Center revelaram que o primeiro evento arrítmico ocorreu três dias após o 9/11. No primeiro mês depois do atentado houve um aumento do risco de 2-3 vezes de taquiarritmias e posteriormente, a taxa de incidência retornou ao normal ${ }^{14}$. Este estudo não foi especificamente feito para investigar CMT, mas as taquiarritmias podem ser consideradas um substituto razoável pelas semelhanças fisiopatológicas das anormalidades da parede cardíaca incitada pelo estresse mental agudo. No rescaldo do terremoto de Chuetsu, o intervalo médio entre terremoto e aparecimento dos sintomas da CMT foi de 2,5 dias; sendo que 11 dos 16 pacientes desenvolveram sintomas no mesmo dia que o terremoto ${ }^{14}$.

Existem poucos dados acerca do tempo transcorrido entre o fator estressor e o início dos sintomas. Os registros mais recentes sobre a síndrome de Takotsubo limitam a relação entre o fator estressor e a ocorrência da síndrome em até uma semana. Mais dados são claramente necessários para determinar se há um padrão da relação temporal entre o estressor e o início dos sintomas ${ }^{10}$.

Embora seja coerente que um estressor emocional ou físico é muitas vezes, mas nem sempre encontrado, não há consistência na definição do que qualifica um evento como estressor. Existe uma enorme variabilidade na descrição desses fatores precipitantes, desde morte de um ente querido até exercícios físicos incomuns ${ }^{14}$.

\section{CONCLUSÃO}

A Cardiomiopatia de Takotsubo deve sempre ser considerada no diagnóstico diferencial da Síndrome coronariana aguda, especialmente quando os sintomas forem precipitados por intenso estresse emocional. Nossos dados revelam que menos da metade dos pacientes tiveram um estressor claramente identificável, quando o viés retrospecto é mínimo. Acreditamos por meio deste estudo que o estresse não é o fator definidor da CMT, levando-se em consideração a inconsistência deste fator em diversos estudos, incluindo o nosso. Pesquisas adicionais, idealmente usando dados de múltiplas instituições e uma amostra maior de pacientes que sofreram de CMT, são necessárias para se melhor determinar e compreender os fatores causais dessa síndrome rara.

\section{REFERENCIAS}

1. Ishikawa K. "Takotsubo" cardiomyopathy. A syndrome characterized by transient left ventricular apical ballooning that mimics the shape of a bottle used for trapping octopus in Japan. Intern Med. 2004; 43(4): 275-6.

2. Prasad A, Lerman A, Rihal CS. Apical ballooning syndrome (Tako-Tsubo or stress cardiomyopathy): a mimic of acute myocardial infarction. Am Heart $\mathrm{J}$. 2008; 155(3):408-17.

3. Kawai S, Kitakabe A, Tomoike H. Guidelines for diagnosis of takotsubo (ampulla) cardiomyopathy. Circ J. 2007; 71(6):990-2.

4. Azzarelli S, Galassi AR, Amico F, Giacoppo M, Argentino V, Tomasello SD et al. Clinical features of transient left ventricular apical ballooning. Am J Cardiol 2006; 98(9):1273-6.

5. El-Battrawy I, Behnes M, Hillenbrand D, Haghi D, Hoffmann U, Papavassiliu T et al. Prevalence, clinical characteristics, and predictors of patients with thromboembolic events in takotsubo cardiomyopathy. Clin Med Insights. Cardiol. 2016; 10:117-22.

6. Park JH, Kang SJ, Song JK, Kim HK, Lim CM, Kang $\mathrm{DH}$ et al. Left ventricular apical ballooning due to severe physical stress in patients admitted to the medical ICU. CHEST 2005; 128(1):296-302.

7. Facciorusso A, Vigna C, Amico C, Lanna P, Troiano G, Stanislao M et al. Prevalence of Tako-Tsubo Syndrome 
among patients with suspicion of acute coronary syndrome referred to our centre. Int J Cardiol. 2009; 134(2):255-9.

8. Lyon AR, Rees PS, Prasad S, Poole-Wilson PA, Harding SE. Stress (Takotsubo) cardiomyopathy: a novel pathophysiological hypothesis to explain catecholamine-induced acute myocardial stunning. Nat Clin Pract Cardiovasc Med. 2008;5(1):22-9

9. Pereira MS, Sarubbi GD, Rodrigues RB, Fontenele MRL, Lemos DRQ. Takotsubo cardiomyopathy associated with sepsis due to Pseudomonas aeruginosa pneumonia. J Health Biol Sci. 2015; 3(4):242-4.

10. Heubach JF, Ravens U, Kaumann AJ. Epinephrine activates both Gs and Gi pathways, but norepinephrine activates only the Gs pathway through human $\beta 2$ adrenoceptors overexpressed in mouse heart. Mol Pharmacol. 2004; 65(5):1313-22.

11. Elesber AA, Prasad A, Bybee KA, Valeti U, Motiei A, Lerman A et al. Transient cardiac apical ballooning syndrome: prevalence and clinical implications of right ventricular involvement. J Am Coll Cardiol. 2006; 47(5):1082-3.

12. Hertting, K., Krause, K., Härle, T., Boczor, S., Reimers, J., \& Kuck, K. H. Transient left ventricular apical ballooning in a community hospital in Germany. Int $\mathbf{J}$ Cardiol. 2006; 112(3):282-8.

13. Bybee KA, Prasad A, Barsness GW, Lerman A, Jaffe AS, Murphy JG et al. Clinical characteristics and thrombolysis in myocardial infarction frame counts in women with transient left ventricular apical ballooning syndrome. Am J Cardiol. 2004; 94(3):343-6.

14. Steinberg JS, Arshad A, Kowalski M, Kukar A, Suma $\mathrm{V}$, Vloka $\mathrm{M}$ et al. Increased incidence of lifethreatening ventricular arrhythmias in implantable defibrillator patients after the World Trade Center attack. J Am Coll Cardiol. 2004; 44(6):1261-4.

\section{CONFLITO DE INTERESSES}

Os autores declaram não haver conflitos de interesse.

\section{AUTOR PARA CORRESPONDÊNCIA}

Márcio da Silva Pereira

marcio7x@hotmail.com

Submetido em 11/07/2017

Aceito em 25/08/2017 